\title{
Ex Post Inequality of Opportunity Comparisons
}

\author{
Marc Fleurbaey, Vito Peragine ${ }^{\dagger}$ and Xavier Ramos ${ }^{\ddagger}$
}

September 20, 2015

\begin{abstract}
In this paper we propose different criteria to rank income distributions according to equality of opportunity. Different from existing ones, our criteria explicitly recognize the interplay between circumstances and effort. We characterize them axiomatically and we compare them with existing criteria; then we propose some scalar measures. We show that our ex post criteria are mostly obtained from "seemingly" ex ante properties. In the second part of the paper we apply our new criteria to measuring inequality of opportunity in Germany. We illustrate our ex-post inequality of opportunity approach based on classes, by means of the German Socio-Economic Panel (SOEP) for the first decade of the 2000s.
\end{abstract}

\section{Introduction}

The equality of opportunity (EOp) literature has proposed a number of criteria and methods to rank outcome distributions on the basis of the inequality of opportunity (IOp) they exhibit. These criteria revolve around the idea of distinguishing fair inequalities, i.e., outcome inequalities due to effort and responsibility, from the unfair inequalities that are instead due to exogenous circusmtances (such as race, gender, parental background). For recent surveys, see Ramos and Van de gaer (2012), Ferreira and Peragine (2015), Roemer and Trannoy (2015).

Two main approaches have been proposed to accomplish such a task: the ex ante approach, where the focus is on the inequalities between individual opportunity sets, and the ex post approach, where the focus is on the outcome inequalities among individuals at the same effort level. Theoretical research has shown the incompatibility between these two approaches (Fleurbaey and Peragine, 2013), thereby signaling that they do capture different aspects of the equality of opportunity ideal. And subsequent empirical research has shown that rankings based on the ex ante and ex post approaches do differ when confronted with real data (see, among others, Checchi et al. 2010 and Aaberge et al. 2011).

\footnotetext{
*University of Princeton.

$\dagger$ University of Bari, vitorocco.peragine@uniba.it

$\ddagger_{\text {Universitat Autonoma de Barcelona. }}$
} 
To be more specific, the canonical model of EOp generally assumes the individual outcome to be a function of two classes of variables, circumstances and effort. Then the ex ante approach proceeds by partitioning the population into types, a type being the set of individuals with the same circumstances. The type is a crucial ingredient of the model: in fact, in the ex ante approach, the type-specific distribution of outcomes, i.e., the distribution conditional on circumstances, is interpreted as the opportunity set available to all and each individual in the type. Hence, the inequality between opportunity sets is captured by measuring inequality between the type-specific outcome distributions. Within this approach, different methodologies have been proposed that try to capture such inequality, the most popular being the one that evaluates the type distribution by a simple statistic, its mean, and therefore compares two distributions by comparing ${ }^{1}$ (say by Lorenz or generalized Lorenz criteria) the respective distribution of the type means. This boils down to comparing the smoothed distributions that are obtained by substituting to each individual income the mean income of the type she belongs to.

While computationally simple and, therefore, often used, this approach can be, and has been in fact, criticized as too simplistic: it ignores the interplay between circumstances and effort. It ignores, in other words, that the impact of circumstances on outcome, which is exactly what one tries to capture when measuring IOp, can be different at different effort levels.

On the other hand, the ex post approach proposes a different partition of the population: into tranches, a tranche being a set of the individuals with the same effort level. Once this partition is made, measuring inequality of opportunity amounts to measuring outcome inequality within each tranche. To declare one distribution better than another one it has to obtain dominance (say by Lorenz or generalize Lorenz criteria) at each and every tranche. This method, proposed and used, among others, by Peragine (2002) and Aaberge et al (2011), is surely correct in principle, however it is also very demanding. A less demanding ex post criterion to rank distributions has been proposed by Roemer (1998): it proposes to look only at the worst off individuals in each tranche. Hence he proposes a maximin rule where the minimum is referred to the lowest position in the tranche distribution.

Our paper is close in spirit to Roemer's proposal. However, we find it too limited to evaluate only the worst off in each tranche. Here we propose an alternative ex post criterion which can be interpreted as a generalization of Roemer's: for our criterion, not only the worst off, but also the second worst off, and the third worst off and so on are taken into account. ${ }^{2}$ More precisely, we define a class as a set of individuals that sit at the same position in their respective tranche distributions. The first class is exactly Roemer's maximand. But, contrary to Roemer, we now consider all classes. Members of the same class, in our construction, have exerted different degrees of responsibility; however, via the effect of the circumstances, they are at the same position in their

\footnotetext{
${ }^{1}$ Here we refer to partial orderings and dominance conditions, but of course indices and complete orderings are also possible and have been defined in the literature.

${ }^{2}$ The idea of extending Roemer's criterion was first proposed by Zapata (2011).
} 
tranche distribution. Our ex post criteria will be based on the idea of reducing the inequality between classes.

In this paper we propose different ex post criteria based on such idea: we characterize them axiomatically and we compare them with existing criteria; then we propose some scalar measures. We show that our ex post criteria are mostly obtained from "seemingly" ex ante properties.

In the second part of the paper we apply our new criteria to measuring inequality of opportunity in Germany. We illustrate our ex-post inequality of opportunity approach based on classes, by means of the German Socio-Economic Panel (SOEP) for the first decade of the 2000s.

Our results show that the class approach does yield different point estimates of inequality of opportunity with respect to the ex ante (type) and the ex post (tranche) approaches, both in absolute levels and as percentage of outcome inequality. However, the ordering of the three approaches crucially depends on the number of types, tranches, and classes. The class approach also reacts differently than the other ex post approach to changes in the number of partitions.

\section{The model}

Each individual is fully described by two sets of traits: circumstances beyond individual control, $\mathbf{c}$, and responsibility characteristics, $e$, called effort. Circumstances and effort belong to finite sets $C=\left\{\mathbf{c}_{1}, \ldots, \mathbf{c}_{\mathbf{n}}\right\}$ and $E=\left\{e^{1}, \ldots, e^{m}\right\}$.

The outcome of interest is generated by a function $g: \Omega \times \Theta \rightarrow \mathbb{R}_{+}$:

$$
x=g(\mathbf{c}, e) .
$$

This model excludes the existence of random components: ${ }^{3}$ for all $i=1, \ldots, n$ and for all $j=1, \ldots, m$, by $x_{i}^{j}$ we denote the outcome generated by circumstances $\mathbf{c}_{i}$ and effort $e^{j}$.

In the general case, we would have different proportions $w_{i}^{j}$ of individuals with outcome $x_{i}^{j}$, such that, with normalization, $\sum_{i=1}^{n} \sum_{j=1}^{m} w_{i}^{j}=1$. However, to simplify the notation, we consider a model in which $w_{i}^{j}=w_{h}^{k}$, for all $i, h=1, \ldots, n$ and for all $j, k=1, \ldots, m$. Moreover we assume full observability of circumstances, effort, and outcome (alternatively, we assume that the effort can be proxied, as in Roemer's percentile approach). Hence our distribution of circumstances, effort and outcomes can be represented by an outcome matrix:

$$
X=\left[\begin{array}{ccc}
x_{1}^{1} & x_{1}^{j} & x_{1}^{m} \\
\cdots & \cdots & \cdots \\
x_{i}^{1} & x_{i}^{j} & x_{i}^{m} \\
\cdots & \cdots & \cdots \\
x_{n}^{1} & x_{n}^{j} & x_{n}^{m}
\end{array}\right]
$$

\footnotetext{
${ }^{3}$ This is consistent with the ex post approach. Inequalities in luck, considered after random factors have struck, are most naturally considered to be part of the circumstances. See Lefranc et al. (2009) for a different model in which luck is treated as a third argument and is not compensated.
} 
Figure 1: Outcome distribution

\begin{tabular}{c|ccc} 
& $e_{1}$ & $e_{2}$ & $e_{3}$ \\
\hline$c_{1}$ & $x_{1,1}$ & $x_{1,2}$ & $x_{1,3}$ \\
$c_{2}$ & $x_{2,1}$ & $x_{2,2}$ & $x_{2,3}$ \\
$c_{3}$ & $x_{3,1}$ & $x_{3,2}$ & $x_{3,3}$
\end{tabular}

Figure 1 represents the distribution of outcome $X$ in a simplified representation with $|\Omega|=3$ and $|\Theta|=3$ and $w_{i, j}=1$ for all $i, j$.

We can identify two different partitions. Each row in a matrix is the outcome vector of a 'type', defined as the set of individuals sharing the same circumstances. Hence, for all $i=1, \ldots, n$, type $i$ is the set of individuals with circumstances $\mathbf{c}_{i}$; the outcome vector of type $i$ is denoted by $X_{i}$ and $\mu\left(X_{i}\right)$ is the average outcome of type $i$. The overall outcome distribution can also be written as $X=\left\{X_{1}, X_{2}, \ldots, X_{n}\right\}$. The second partition is defined by effort: each group in this partition is called a 'tranche', and includes all individuals that exerted the same effort. For all $j=1, \ldots, m$, tranche $j$ is the set of individuals who have chosen effort $e^{j}$; the tranche $j$ outcome distribution is represented by the column $j$ in the matrix and is denoted by $X^{j} ; \mu\left(X^{j}\right)$ is the average outcome of tranche $j$. Therefore the outcome distribution can also be written as $X=\left\{X^{1}, X^{2}, \ldots, X^{m}\right\}$.

Let $D$ be the set of admissible distributions. Moreover let $D^{>}$be the set of distributions characterized by vector dominance (denoted by $\geqslant$ ) of the type distributions: $D^{>}=:\left\{X \in D: X_{i} \geqslant X_{i+1}, \forall i\right\}$.

Starting from the original distribution $X \in D$, we can construct a new distribution $X^{>}$by permuting each tranche distribution so that now $X^{>} \in D^{>}$. Hence, in this transformed distribution the rows dominate each other: we call the rows of this new distribution "classes" (by a slight abuse of terminology, we also call class the set of individuals whose outcomes constitute a class distribution) and we denote them by $X_{i}^{>}$. We assume they are ordered such that $X_{i}^{>} \geqslant X_{i+1}^{>}, \forall i=1, \ldots, n-1$. Hence we have a further partition of the original distribution $X$ into classes, so that $X^{>}$can be written as: $X^{>}=\left\{X_{1}^{>}, X_{2}^{>}, \ldots, X_{n}^{>}\right\}$.

Starting from an original distribution $X \in D$, we can also construct a new distribution $X^{+}$by permuting each type distribution so that $\left(x^{+}\right)_{i}^{j} \leq\left(x^{+}\right)_{i}^{j+1}$ for all $j=1, \ldots, m-1$. This is automatically obtained when effort is measured via Roemer's identification assumption, i.e., by the rank in the distribution of the type.

Members of the same class, in our construction, have exerted different degrees of responsibility; however, via the effect of the circumstances, they sit at the same position in their respective tranche distribution. Hence we take the position of individuals in the respective tranche distribution as an interpersonal 
comparable measure of the impact of circumstances. Those who are the worst off in their tranches, i.e., members of the lowest class, share the feature that they are the most disadvantaged by circumstances - even if they belong to different types. Those who are at the top in their tranches, i.e., members of the highest class, are the most advantaged by circumstances. And so on for the intermediate classes. In this way we are able to capture the effect of circumstances in a comparable way across individuals, given their level of effort.

For a given distribution $X \in D$, let $\mathbf{1}_{m}$ be the unit vector of size $m$, we can define the following smoothed distribution, which contains only inequality between types and not within types:

$$
\boldsymbol{\mu}(X):=\left(\mathbf{1}_{m} \mu\left(X_{1}\right), \ldots, \mathbf{1}_{m} \mu\left(X_{n}\right)\right) .
$$

Moreover, let $\succeq_{F O D}, \succeq_{L} \succeq_{G L}$ denote the first order dominance, Lorenz and generalized Lorenz dominance respectively.

We are interested in ranking members of $D$ according to equality of opportunity.

\section{$3 \quad$ Equality of opportunity partial orderings}

The existing literature has explored two main approaches to measuring opportunity inequality: the ex ante and the ex post approach.

Ex post opportunity inequality: There is EOp if all those who exert the same effort have the same outcome. Inequality of opportunity decreases if outcome inequality decreases among the individuals at the same degree of effort.

The ex post EOp focuses on the effect of circumstances on individual outcomes. Hence, to implement such approach, one needs to identify the effort of individuals: this is why we call it the ex post approach.

This is the approach proposed by Roemer $(1993,1998)$ and Fleurbaey $(2008)$.

On the contrary, the ex ante approach focuses on the effect of circumstances on the types' outcomes, as summarized by the type income distribution, which is interpreted as the individual opportunity set. The utilitarian version focuses exclusively on the type means. Hence, to implement such approach, one does not need to observe the effort, but only the outcome distribution of each type.

The ex ante approach.

There is EOp if the opportunity sets have the same value, regardless of the circumstances. Inequality of opportunity decreases if inequality between individual opportunity sets decreases.

The ex ante approach puts special emphasis on the differences in the outcome prospects for classes of individuals with identical circumstances. Accordingly, it focuses on inequality between types. This is the approach proposed, in different frameworks, by Van de gaer (1993) and Kranich (1996).

Hence, the ex ante approach is more focused on inequalities between social groups. In contrast, the ex post approach scrutinizes individual outcome inequalities due to their belonging to social groups.

More precisely, the existing literature has proposed and characterized, in a variety of settings, the following partial orderings. 
1) Ex ante Utilitarian: ${ }^{4}$ For all $X, Y \in D, X \succeq Y$ if and only if $\sum_{i=1}^{k} \mu\left(X_{i}\right) \geq$ $\sum_{i=1}^{k} \mu\left(Y_{i}\right) \forall k=1, \ldots, n$ (Peragine 2002).

2) Ex ante Pareto: For all $X, Y \in D, X \succeq Y$ if and only if $\sum_{i=1}^{k} X_{i} \geq_{F O D}$ $\sum_{i=1}^{k} Y_{i} \forall k=1, \ldots, n$ (Peragine and Serlenga 2008).

3) Ex post : For all $X, Y \in D, X \succeq Y$ if and only if $X^{j} \geq_{G L} Y^{j} \forall j=1, \ldots, m$ (Peragine 2002, see also Aaberge et al 2011).

Scalar inequality measures naturally associated to rankings 1 and 3 have also been proposed and applied in empirical works (see Checchi and Peragine 2010, Ferreira and Gignoux 2011, and Aaberge et al 2011).

The aim of this paper is to further explore the ex post approach and to propose new criteria coherent with such view. We propose the following equality of opportunity partial orderings:

Definition 1 Utilitarian ex post : For all $X, Y \in D, X \succeq_{U-E O P} Y$ if and only if $\boldsymbol{\mu}\left(X^{>}\right) \succeq_{G L} \boldsymbol{\mu}\left(Y^{>}\right)$.

Definition 2 Anonymous ex post: For all $X, Y \in D, X \succeq_{A-E O P} Y$ if and only if $\sum_{i=1}^{k} X_{i}^{>+} \geq \sum_{i=1}^{k} Y_{i}^{>+} \forall k=1, \ldots, n$

Definition 3 Non anonymous ex post: For all $X, Y \in D, X \succeq_{N A-E O P} Y$ if and only if $\sum_{i=1}^{k} X_{i}^{>} \geq \sum_{i=1}^{k} Y_{i}^{>} \forall k=1, \ldots, n$

Definition 4 Egalitarian ex post: For all $X, Y \in D, X \succeq_{E-E O P} Y$ if and only if $\sum_{i=1}^{k} X_{i}^{>+} \succeq_{G L} \sum_{i=1}^{k} Y_{i}^{>+} \forall k=1, \ldots, n$

The criterion $\succeq_{U-E O P}$ is a standard generalized Lorenz dominance applied to the smoothed distributions obtained from $X$ and $Y$ after partitioning such distributions into classes: $\left(\mathbf{1}_{N_{1}} \mu\left(X_{1}^{>}\right), \ldots, \mathbf{1}_{N n} \mu\left(X_{n}^{>}\right)\right) \geq_{G L}\left(\mathbf{1}_{N_{1}} \mu\left(Y_{1}^{>}\right), \ldots, \mathbf{1}_{N n} \mu\left(Y_{n}^{>}\right)\right)$. It is based on a judgment of neutrality with respect to the outcome inequality within a class.

The criterion $\succeq_{A-E O P}$ is a sequential test of first order dominance. For each of the two distributions $X, Y$, start from the lowest class, then add the second, then the third, and so on, until one obtains the overall distribution. At each step check for first order dominance of one distribution over the other. ${ }^{5}$ It is based on a position of indifference with respect to the relationship between effort and outcome. ${ }^{6}$

The criterion $\succeq_{N A-E O P}$ is a sequential test of vector dominance. For each of the two distributions $X, Y$, start from the lowest class, then add the second, and so on. At each step, check for dominance of one distribution over the other. This "agnostic" criterion is compatible with all views about the link between effort and outcomes.

\footnotetext{
${ }^{4}$ An agnostic (non-utilitarian) version of the ex ante equality of opportunity criterion would be the following: For all $X, Y \in D, X \succeq Y$ if and only if $\sum_{i=1}^{k} X_{i} \geqslant \sum_{i=1}^{k} Y_{i} \forall k=1, \ldots, n$.

${ }^{5}$ Notice that the rankings in definitions 1 and 2 coincide respectively to the rankings 1 ) and 2) if $X, Y \in D^{>}$.

${ }^{6}$ Notice that the rankings in definitions 1 and 2 coincide respectively to the rankings 1 ) and 2) if $X, Y \in D^{>}$.
} 
The difference between $\succeq_{A-E O P}$ and $\succeq_{N A-E O P}$ is in the anonymity axiom: this becomes relevant in an empirical application, because the ordering by effort, in a given class, can be different from the ordering according to incomes.

Notice that, in the case of using Roemer's identification axiom for the identification of effort, there is no difference between anonymous and non anonymous, as the ordering within a class is the same either according to income or effort.

The criterion $\succeq_{E-E O P}$ is a sequential test of second order dominance. This dominance relation can also be interpreted as generalized Lorenz dominance within each tranche.

Notice also the following implications: For all $X, Y \in D$,

$$
X \succeq_{N A-E O P} Y \Rightarrow X \succeq_{A-E O P} Y \Rightarrow X \succeq_{E-E O P} Y \Rightarrow X \succeq_{U-E O P} Y .
$$

\section{The axioms}

Consider the following axioms that can be imposed on the relation $\succeq$ defined on $D$. The first one says if each tranche has a weakly better distribution, then the whole matrix is weakly preferable.

Axiom 5 Dominance (DOM). For all $X, Y \in D$, if for all tranches $j=$ $1, \ldots, m,\left[X^{j}, X^{j}, \ldots, X^{j}\right] \succeq\left[Y^{j}, Y^{j}, \ldots, Y^{j}\right]$ then $X \succeq Y$. If $\left[X^{j}, X^{j}, \ldots, X^{j}\right] \succ$ $\left[Y^{j}, Y^{j}, \ldots, Y^{j}\right]$ for at least one $j$, then $X \succ Y$.

Next we introduce an axiom of anonymity, requiring social indifference with respect to permutations between the types;

Axiom 6 Anonymity between types (ANB). For all $X, Y \in D^{>}$, for all permutations $\pi$ over $\{1, \ldots, n\}$, if for all types $i=1, \ldots, n, Y_{i}=X_{\pi(i)}$ then $Y \sim X$

The next axioms are all imposed on ordered distributions, i.e., members of $D^{>}$. Moreover, all the axioms below, except for the transfer axioms, seem to follow from an ex ante approach, in that they do not start from the identification of the effort.

The first is a standard monotonicity axiom, stating that an income increment increases social welfare.

Axiom 7 Monotonicity (MON) For all $X, Y \in D^{>}$, if for all types $s \neq i$, $X_{s}=Y_{s}$, for all $t \neq j, x_{i}^{t}=y_{i}^{t}$, and $y_{i}^{j}=x_{i}^{j}+\epsilon$, with $\epsilon>0$, then $Y \succ X$.

Next we introduce an axiom concerned with permutations within classes.

Axiom 8 Anonymity within classes $(\boldsymbol{A N} \boldsymbol{N})$. For all $X, Y \in D^{>}$, if for all classes $i=1, \ldots, n$, there is a permutation $\pi_{i}$ over $\{1, \ldots, m\}$ such that for all tranches $j=1, \ldots, m, y_{i}^{j}=x_{i}^{\pi_{i}(j)}$ then $Y \sim X$. 
The next axiom is an expression of the reward principle, in its utilitarian version. It considers a special case in which the two distributions under consideration have, for each type, the same mean income. In this case the two distributions are declared indifferent.

Axiom 9 Inequality neutrality within classes (INW). For all $X, Y \in$ $D^{>}$, if for all classes $i=1, \ldots, n, \mu\left(X_{i}\right)=\mu\left(Y_{i}\right)$, then $Y \sim X$.

The next two axioms are concerned with progressive transfers: the former with transfers between classes (from higher to lower types), the latter with transfers within classes (from richer to poorer individuals).

Axiom 10 Inequality aversion between classes (IAB). For all $X, Y \in$ $D^{>}$, for all classes $i<h$, all tranches $j$, if for all classes $s \neq h, i, X_{s}=Y_{s}$, for all $t \neq j, x_{i}^{t}=y_{i}^{t}$ and $x_{h}^{t}=y_{h}^{t}$, and $y_{h}^{j}=x_{h}^{j}-\epsilon \geq x_{i}^{j}+\epsilon=y_{i}^{j}$ with $\epsilon>0$, then $Y \succ X$.

Similarly, one can have progressive transfers within classes (from richer to poorer tranches).

Axiom 11 Inequality aversion within classes (INW). For all $X, Y \in$ $D^{>}$, if for all classess $\neq h, X_{s}=Y_{s}$, for all $t \neq i, j, x_{h}^{t}=y_{h}^{t}$, and $y_{h}^{j}=x_{h}^{j}-\epsilon \geq$ $x_{h}^{i}+\epsilon=y_{h}^{i}$ with $\epsilon>0$, then $Y \succ X$.

Lastly, composite transfers involving a progressive transfer within a class combined with the opposite regressive transfer within a higher class are condoned by the following axiom. Note that such composite transfers can also be described as the combination of a progressive transfer between classes combined with the opposite transfer between the same classes at more advantaged tranches. In both cases, the motivation is the idea of decreasing inequality aversion (principle of diminishing transfers).

Axiom 12 Diminishing inequality aversion within classes (DIAW). For all $X, Y \in D^{>}$, all classes $h<i$, all tranches $j, k$, if for all types $s \neq h, i$, all tranches $t \neq j, k, x_{s t}=y_{s t}$, and if $x_{h}^{j}=y_{h}^{j}-\epsilon \geq y_{h}^{k}+\epsilon=x_{h}^{k}$ and $x_{i}^{j}+\epsilon=$ $y_{i}^{j} \geq y_{i}^{k}=x_{i}^{k}-\epsilon$ with $\epsilon>0$, then $Y \succ X$.

\section{The results}

Although the axioms above seem to endorse an ex ante approach, the next results show that they are instead able to characterize ex post criteria. This is because they apply only on the subdomain $D^{>}$.

Let us say that $\succeq$ is an extension $\succeq^{*}$ if, for all $X, Y \in D, X \succeq^{*} Y$ implies $X \succeq Y$ and $X \succ^{*} Y$ implies $X \succ Y$.

We obtain the following characterizations.

Proposition 13 If $\succeq$ satisfies DOM, ANB, INW, IAB and MON then $\succeq$ is an extension $\succeq_{U-E O P}$. 
Proof. We first prove a Lemma.

Lemma 1: $A N B$ and DOM imply Anonymity within tranches, defined as follows: For all $X, Y \in D$, if for all tranches $j=1, \ldots, m$, there is a permutation $\pi^{j}$ over $\{1, \ldots, n\}$ such that for all types $i=1, \ldots, n, y_{i}^{j}=x_{\pi^{j}(i)}^{j}$ then $Y \sim X$.

\section{Proof.}

Consider $X$ and $Y$ such that for all $j=1, \ldots, m$, there is a permutation $\pi^{j}$ such that for all $i=1, \ldots, n, y_{i}^{j}=x_{\pi^{j}(i)}^{j}$. Take column (tranche) $j$ from $X$ and construct the clone $j$ matrices $X\langle j\rangle=\left[X^{j}, X^{j}, \ldots, X^{j}\right]$ and $Y\langle j\rangle=$ $\left[Y^{j}, Y^{j}, \ldots, Y^{j}\right]$. We will show that for all $j, Y\langle j\rangle \sim X\langle j\rangle$. By DOM, this will imply $Y \sim X$.

Let $X\left\langle j, \pi^{j}\right\rangle$ be obtained by permuting types in $X\langle j\rangle$ according to $\pi^{j}$ : for all $i, X\left\langle j, \pi^{j}\right\rangle_{i}=X\langle j\rangle_{\pi^{j}(i)}$. By ANB $X\left\langle j, \pi^{j}\right\rangle \sim X\langle j\rangle$. (Note that both matrices belong to $D^{>}$.)

Observe that $Y^{j}=X\left\langle j, \pi^{j}\right\rangle^{j}$. Therefore, one has $Y\langle j\rangle=X\left\langle j, \pi^{j}\right\rangle$, implying $Y\langle j\rangle \sim X\langle j\rangle$.

QED.

We can now turn to the proof of the theorem.

By Anonymity within tranches we can transform any distribution $X \in D$ into a distribution $X^{>} \in D^{>}$.

Hence now we can apply the axioms ANB, INW, IAB and MON.

The argument relies on the following steps:

1) If $\succcurlyeq$ satisfies $I N W$ then $X \sim \boldsymbol{\mu}(X)$ and $Y \sim \boldsymbol{\mu}(Y)$.

2) If $\succcurlyeq$ satisfies $M O N$ and $I A B$, then $\boldsymbol{\mu}(X) \succcurlyeq \boldsymbol{\mu}(Y)$ if $\left(\mu\left(X_{1}\right), \ldots, \mu\left(X_{n}\right)\right) \succcurlyeq_{G L}$ $\left(\mu\left(Y_{1}\right), \ldots, \mu\left(Y_{n}\right)\right)$ and $\boldsymbol{\mu}(X) \succ \boldsymbol{\mu}(Y)$ if $\left(\mu\left(X_{1}\right), \ldots, \mu\left(X_{n}\right)\right) \succ_{G L}\left(\mu\left(Y_{1}\right), \ldots, \mu\left(Y_{n}\right)\right)$.

We now prove each step in turn.

1) Starting from a distribution $X=\left(X_{1}, \ldots, X_{n}\right)$, by $I N W$ we obtain that the smoothed distribution $\boldsymbol{\mu}(X)=\left(\mu_{1} \mathbf{1}_{m}, \ldots, \mu_{n} \mathbf{1}_{m}\right)$ satisfies $X \sim \boldsymbol{\mu}(X)$.

2) We are considering distributions such that $\mu_{i} \leq \mu_{i+1}$ for all $i=1, \ldots, n-1$.

We now show that if $\left(\mu\left(X_{1}\right), \ldots, \mu\left(X_{n}\right)\right) \succ_{G L}\left(\mu\left(Y_{1}\right), \ldots, \mu\left(Y_{n}\right)\right)$ then one can derive $\boldsymbol{\mu}(X)$ from $\boldsymbol{\mu}(Y)$ by a finite sequence of between-type transfers and/or increments. This shows that for any $\succcurlyeq$ satisfying $M O N$ and $I A B$, then $\boldsymbol{\mu}(X) \succ \boldsymbol{\mu}(Y)$ if $\left(\mu\left(X_{1}\right), \ldots, \mu\left(X_{n}\right)\right) \succ_{G L}\left(\mu\left(Y_{1}\right), \ldots, \mu\left(Y_{n}\right)\right)$. (Note that when $\left(\mu\left(X_{1}\right), \ldots, \mu\left(X_{n}\right)\right) \sim_{G L}\left(\mu\left(Y_{1}\right), \ldots, \mu\left(Y_{n}\right)\right)$, necessarily they are equal, implying $\boldsymbol{\mu}(X) \sim \boldsymbol{\mu}(Y)$.)

Let us write $\boldsymbol{\mu}(Y)=\left(y_{1} \mathbf{1}_{m}, \ldots, y_{n} \mathbf{1}_{m}\right)$ and $\boldsymbol{\mu}(X)=\left(x_{1} \mathbf{1}_{m}, \ldots, x_{n} \mathbf{1}_{m}\right)$. We now adapt the Hardy-Littlewood-Polya (HLP) argument to this special case.

Select the lowest $j$ such that $x_{j}<y_{j}$ (if there is none, then $\boldsymbol{\mu}(X) \succ \boldsymbol{\mu}(Y)$ by MON), and the greatest $i<j$ such that $x_{i} \neq y_{i}$. Necessarily $x_{i}>y_{i}$. Let $\delta=\min \left\{y_{j}-x_{j}, x_{i}-y_{i}\right\}$.

Now, starting from $Y$, all individuals from type $j$ give $\delta$; all individuals from type $i$ receive $\delta$. This yields a new distribution $Y^{1}$ such that: $y_{i}^{1}=y_{i}+\delta, y_{j}^{1}=$ $y_{j}-\delta$, and $y_{k}^{1}=y_{k} \forall k \neq i, j$. One has $y_{i}+\delta \leq x_{i}, y_{j}-\delta \geq x_{j}$, with at least one equality.

For $Y^{1}$ it is still the case that $y_{k}^{1} \leq y_{k+1}^{1}$ for all $k=1, \ldots, n-1$, i.e., $Y^{1} \in D^{>}$. In particular, $y_{i}^{1} \leq x_{i} \leq x_{i+1}$ and $x_{i+1}=y_{i+1}^{1}$ if $i+1<j$, and $x_{i+1} \leq y_{i+1}^{1}$ 
if $i+1=j$. Similarly, $y_{j}^{1} \geq x_{j} \geq x_{j-1}$, and $x_{j-1}=y_{j-1}^{1}$ if $j-1>i$, and $x_{j-1} \geq y_{j-1}^{1}$ if $j-1=i$. By IAB, one has $Y^{1} \succ \boldsymbol{\mu}(Y)$.

We now check that $\boldsymbol{\mu}(X) \succeq_{G L} Y^{1}$, i.e., $\forall k=1, \ldots, n: \sum_{t=1}^{k} x_{t} \geq \sum_{t=1}^{k} y_{t}^{1}$.

For $k<i: \sum_{t=1}^{k} x_{t}=\sum_{t=1}^{k} y_{t}^{1}$ because $x_{t}=y_{t}^{1}$ for all $t$.

For $k=i: \sum_{t=1}^{i} x_{t}=\sum_{t=1}^{i-1} x_{t}+x_{i}=\sum_{t=1}^{i-1} y_{t}^{1}+x_{i} \geq \sum_{t=1}^{i-1} y_{t}^{1}+y_{i}^{1}=$ $\sum_{t=1}^{i} y_{t}^{1}$.

For $i<k<j: \sum_{t=1}^{k} x_{t}=\sum_{t=1}^{i} x_{t}+\sum_{t=i+1}^{k} x_{t} \geq \sum_{t=1}^{i} y_{t}^{1}+\sum_{t=i+1}^{k} x_{t}=$ $\sum_{t=1}^{k} y_{t}^{1}$.

For $k \geq j: \sum_{t=1}^{k} x_{t} \geq \sum_{t=1}^{k} y_{t}=\sum_{t=1}^{k} y_{t}^{1}$.

Iterating, after at most $n-1$ steps, one reaches $\boldsymbol{\mu}(X)$, thereby proving by transitivity that $\boldsymbol{\mu}(X) \succ \boldsymbol{\mu}(Y)$.

QED

Proposition 14 If $\succeq$ satisfies DOM, ANB, ANW, IAB and MON, then $\succeq$ is an extension $\succeq_{A-E O P}$.

Proof. The proof is articulated in the following steps:

1) Starting from a distribution $X \in D$, by application of DOM and ANB, and by applying Lemma 1, we can obtain a new distribution $X^{>} \sim X \in D^{>}$.

2) By applying ANW to $X^{>}$we order the incomes increasingly, thereby obtaining a new distribution $X^{>+} \sim X^{>} \sim X$. Note that $X^{>+} \in D^{>}$, because if two vectors $x, y$ satisfy $x \geq y$, then reordering their components in increasing order, yielding $x^{+}, y^{+}$, one still has $x^{+} \geq y^{+}$. (This is shown as follows. There is no loss in generality in assuming $y=y^{+}$. One has $x \geq y^{+}$. One goes from $x$ to $x^{+}$by a succession of transpositions that reorder pairs of components by increasing order. After each transposition, the vector inequality is preserved because it transforms a configuration $\begin{gathered}x_{t}>x_{s} \\ y_{k}^{+} \leq y_{k+h}^{+}\end{gathered}$where $x_{s} \geq y_{k+h}^{+}$into $\begin{gathered}x_{s}<x_{t} \\ y_{k}^{+} \leq y_{k+h}^{+}\end{gathered}$ where obviously $x_{s} \geq y_{k}^{+}$and $x_{t} \geq y_{k+h}^{+}$.)

3) Assume that $\sum_{i=1}^{k} X_{i}^{>+}=\sum_{i=1}^{k} Y_{i}^{>+} \forall k=1, \ldots, n$. This implies that $X^{>+}=Y^{>+}$, therefore $X \sim Y$ by the previous steps.

4) Assume that $\sum_{i=1}^{k} X_{i}^{>+} \geq \sum_{i=1}^{k} Y_{i}^{>+} \forall k=1, \ldots, n$, with strict inequality for at least one $k$. This means that for each $j=1, \ldots, m, X^{>+j} \succeq_{G L} Y^{>+j}$, with strict GL dominance for at least one $j$. By the HLP argument, each $X^{>+j}$ can be obtained from $Y^{>+j}$ by a series of increments and/or Pigou-Dalton transfers. The former are condoned by MON and the latter by IAB. Therefore, by DOM $X^{>+} \succ Y^{>+}$. By transitivity, $X \succ Y$.

QED

Proposition 15 If $\succeq$ satisfies DOM, ANB, IAB and MON, then $\succeq$ is an extension of $\succeq_{N A-E O P}$.

Proof. The proof is the same as the previous one, except that step 2 is no longer needed, and steps 3 and 4 are modified as follows: 
3) Assume that $\sum_{i=1}^{k} X_{i}^{>}=\sum_{i=1}^{k} Y_{i}^{>} \forall k=1, \ldots, n$. This implies that $X^{>}=Y^{>}$, therefore $X \sim Y$ by step 1 .

4) Assume that $\sum_{i=1}^{k} X_{i}^{>} \geq \sum_{i=1}^{k} Y_{i}^{>} \forall k=1, \ldots, n$, with strict inequality for at least one $k$. This means that for each $j=1, \ldots, m, X^{>j} \succeq_{G L} Y^{>j}$, with strict GL dominance for at least on $j$. By the HLP argument, each $X^{>j}$ can be obtained from $Y^{>j}$ by a series of increments and/or Pigou-Dalton transfers. The former are condoned by MON and the latter by IAB. Therefore, by DOM, $X^{>} \succ Y^{>}$. By transitivity, $X \succ Y$.

QED

Proposition 16 If $\succeq$ satisfies DOM, ANB, IAW, IAB, ANW, DIAW and $M O N$, then $\succeq$ is an extension of $\succeq_{E-E O P}$.

Proof. 1) By DOM, ANB, ANW, one can replace $X, Y$ by $X^{>+}, Y^{>+}$.

2) Let $\sum_{i=1}^{k} X_{i}^{>+} \succeq_{G L} \sum_{i=1}^{k} Y_{i}^{>+} \forall k=1, \ldots, n$. Note that if $\sum_{i=1}^{k} X_{i}^{>+} \sim_{G L}$ $\sum_{i=1}^{k} Y_{i}^{>+} \forall k=1, \ldots, n$, then $X^{>+}=Y^{>+}$.

3) Focus on the first step of the sequential procedure: $X_{1}^{>+} \succeq_{G L} Y_{1}^{>+}$. By the HLP argument, $X_{1}^{>+}$is obtained from $Y_{1}^{>+}$by a sequence of increments and/or Pigou-Dalton transfers, condoned by MON and IAW.

4) Consider $X_{1}^{>+}+X_{2}^{>+} \succeq_{G L} Y_{1}^{>+}+Y_{2}^{>+} \cdot X_{2}^{>+}$is obtained from $Y_{2}^{>+}$ by increments and/or Pigou-Dalton transfers, but also by decrements and/or regressive transfers. Some restrictions apply to the decrements and regressive transfers in order to preserve $X_{1}^{>+}+X_{2}^{>+} \succeq_{G L} Y_{1}^{>+}+Y_{2}^{>+}$. The decrements must (possibly partly) undo the increments received by $X_{1}^{>+}$, and the regressive transfers must (possibly partly) undo the progressive transfers generating $X_{1}^{>+}$. Now, a combination of increment to $Y_{1}^{>+}$and decrement to $Y_{2}^{>+}$(that undoes the former, therefore operates on the same tranche) is condoned by IAB. And a combination of progressive transfer on $Y_{1}^{>+}$and regressive transfer on $Y_{2}^{>+}$ (that undoes the former, therefore operates on the same pair of tranches) is condoned by DIAW.

5) By iteration, if $\sum_{i=1}^{k} X_{i}^{>+} \succeq_{G L} \sum_{i=1}^{k} Y_{i}^{>+} \forall k=1, \ldots, s$, and if up to $s-1$, $X_{k}^{>+}$is obtained from $Y_{k}^{>+}$by increments (MON), progressive transfers (IAW), decrements involved in progressive transfers to lower types (IAB), or regressive transfers involved in composite transfers involving lower types (DIAW), then $X_{s}^{>+}$is obtained from $Y_{s}^{>+}$by the same operations. Note that it is impossible, at a higher type, to undo a progressive transfer or a composite transfer between lower types, because such transfers just preserve the sum $\sum_{i=1}^{k} Y_{i}^{>+}$. Therefore the reasoning of step 4 applies, referring only to decrements undoing increments at lower types and regressive transfers undoing progressive transfers at lower types.

The adaptation of these arguments to introduce strict inequalities and strict GL dominance are omitted here.

QED 


\section{Opportunity inequality indexes}

In this section we propose inequality and welfare complete orderings which are naturally associated to the utilitarian ex post partial ordering defined above, which can be used in empirical applications. First define the matrices $\boldsymbol{\mu}_{B}\left(X^{>}\right)$ and $\boldsymbol{\mu}_{W}\left(X^{>}\right)$as follows

$$
\begin{gathered}
\boldsymbol{\mu}_{B}\left(X^{>}\right)=\left[\begin{array}{ccc}
\mu_{1} & \ldots & \mu_{1} \\
\mu_{2} & \cdots & \mu_{2} \\
\vdots & \vdots & \vdots \\
\mu_{n} & \cdots & \mu_{n}
\end{array}\right] \\
\boldsymbol{\mu}_{W}\left(X^{>}\right)=\left[\begin{array}{cccc}
\frac{x_{11}}{\mu_{1}} & \frac{x_{12}}{\mu_{1}} & \ldots & \frac{x_{1 m}}{\mu_{1}} \\
\frac{x_{21}}{\mu_{2}} & \frac{x_{22}}{\mu_{2}} & \ldots & \frac{x_{2 m}}{\mu_{2}} \\
\vdots & \vdots & \ddots & \vdots \\
\frac{x_{n 1}}{\mu_{n}} & \frac{x_{n 2}}{\mu_{n}} & \ldots & \frac{x_{n m}}{\mu_{n}}
\end{array}\right]
\end{gathered}
$$

$\boldsymbol{\mu}_{B}\left(X^{>}\right)$eliminates the within class inequality, hence contains only inequality between classes, while $\boldsymbol{\mu}_{W}\left(X^{>}\right)$eliminates the between class inequality, hence contains only inequality within classes.

Consider a Lorenz consistent inequality index $I: D \rightarrow \Re_{+}$. Then we can define a measure of opportunity inequality as

$$
I_{U-E O P}=I\left(\boldsymbol{\mu}_{B}\left(X^{>}\right)\right)
$$

$I\left(\boldsymbol{\mu}_{B}\left(X^{>}\right)\right)$captures the inequality between classes. A relative version of it would be

$$
I_{U-E O P}^{R}=\frac{I\left(\boldsymbol{\mu}_{B}\left(X^{>}\right)\right)}{I(X)}
$$

If we use a path independent index (Foster and Shneyerov 2000), we obtain the following decomposition

$$
I(X)=I\left(\boldsymbol{\mu}_{B}\left(X^{>}\right)\right)+I\left(\boldsymbol{\mu}_{W}\left(X^{>}\right)\right)
$$

The interpretation is as follows: total inequality is given by the sum of opportunity inequality (inequality between classes) and effort inequality (inequality within classes).

It is also possible to define normative indexes. Let us define the following normative smoothed distributions $\mathbf{E D E}\left(X^{>}\right), \mathbf{E D E}_{B}\left(X^{>}\right)$and $\mathbf{E D E}_{W}\left(X^{>}\right)$ as

$$
\operatorname{EDE}\left(X^{>}\right)=\left[\begin{array}{ccc}
E D E & \ldots & E D E \\
E D E & \ldots & E D E \\
\vdots & \vdots & \vdots \\
E D E & \ldots & E D E
\end{array}\right]
$$


such that

$$
\begin{gathered}
W(X)=W\left(\mathbf{E D E}\left(X^{>}\right)\right) \\
\operatorname{EDE}_{B}\left(X^{>}\right)=\left[\begin{array}{ccc}
E D E_{1} & \ldots & E D E_{1} \\
E D E_{2} & \ldots & E D E_{2} \\
\vdots & \vdots & \vdots \\
E D E_{n} & \ldots & E D E_{n}
\end{array}\right]
\end{gathered}
$$

such that for all $i=1, \ldots, n$,

$$
\begin{gathered}
W\left(\left[\begin{array}{c}
X_{i}^{>} \\
\vdots \\
X_{i}^{>}
\end{array}\right]\right)=W\left(\left[\begin{array}{c}
E D E_{i} \\
\vdots \\
E D E_{i}
\end{array}\right]\right) ; \\
\mathbf{E D E}_{W}\left(X^{>}\right)=\left[\begin{array}{ccc}
E D E^{1} & \ldots & E D E^{m} \\
E D E^{1} & \ldots & E D E^{m} \\
\vdots & \ddots & \vdots \\
E D E^{1} & \ldots & E D E^{m}
\end{array}\right]
\end{gathered}
$$

such that for all $j=1, \ldots, m$,

$$
W\left(\left[\begin{array}{lll}
X^{>j} & \cdots & X^{>j}
\end{array}\right]\right)=W\left(\left[\begin{array}{lll}
E D E^{j} & \cdots & E D E^{j}
\end{array}\right]\right) .
$$

Given the same level of social welfare, $\operatorname{EDE}\left(X^{>}\right)$eliminates the overall inequality, $\mathbf{E D E}_{B}\left(X^{>}\right)$eliminates the within class inequality, hence contains only inequality between classes, while $\mathbf{E D E}_{W}\left(X^{>}\right)$eliminates the between class inequality, hence contains only inequality within classes.

In the definition of the various $E D E$ s we can assume different degrees of aversion to opportunity inequality.

Then we can propose the following inequality indexes:

$$
I_{O}=1-\frac{\mu\left(\mathbf{E D E}\left(X^{>}\right)\right)}{\mu\left(\mathbf{E D E}_{B}\left(X^{>}\right)\right)}
$$

which captures the cost of inequality between classes, and hence can be interpreted as an index of ex post inequality of opportunity;

$$
I_{E}=1-\frac{\mu\left(\mathbf{E D E}_{B}\left(X^{>}\right)\right)}{\mu(X)}
$$

which capture the cost of inequality within classes, and hence can be interpreted as an index of inequality of effort;

$$
I=1-\frac{\mu\left(\operatorname{EDE}\left(X^{>}\right)\right)}{\mu(X)}
$$

which captures the cost of total inequality. 
Using the multiplicative decomposition properties of the indexes, we obtain the following decomposition:

$$
I=I_{O}+I_{E}+I_{O} I_{E}
$$

Alternatively, we can define the following indices:

$$
I_{O}^{\prime}=1-\frac{\mu\left(\mathbf{E D E}_{W}\left(X^{>}\right)\right)}{\mu(X)}
$$

which captures the cost of inequality between classes, and hence can be interpreted as an index of ex post inequality of opportunity;

$$
I_{E}^{\prime}=1-\frac{\mu\left(\mathbf{E D E}\left(X^{>}\right)\right)}{\mu\left(\mathbf{E D E}_{W}\left(X^{>}\right)\right)}
$$

which capture the cost of inequality within classes, and hence can be interpreted as an index of inequality of effort.

And we obtain the following decomposition:

$$
I=I_{O}^{\prime}+I_{E}^{\prime}+I_{O}^{\prime} I_{E}^{\prime}
$$

\section{$7 \quad$ Ex post inequality of opportunity in Germany}

To illustrate our novel class ex-post approach to inequality of opportunity, we propose two empirical exercises. First we compare the three approaches (types, tranches and classes) using data for a single year, 2000. In this first exercise we also examine the robustness of our estimates to changing the number of tranches and classes. A second exercise examines whether different approaches yield different inequality of opportunity orderings over three points in time, 2000, 2005, and 2010.

\subsection{Data}

We illustrate our ex-post inequality of opportunity approach based on classes, by means of the German Socio-Economic Panel ${ }^{7}$ (SOEP) for the first decade of the 2000s. This data set is representative of the German population, and contains information on a wide range of personal and household information, and also a set of questions on family background, personality traits or anthropometric measures, over which types can be defined. Notwithstanding this, due to small cell sizes, we have to dispose of large parts of this information and define types over a set of three variables only: (i) whether the individual is a men or a women, (ii) whether the individual is younger or older than 40, and (iii) whether the highest educational attainment of the parents is higher or lower than secondary education. We thus have 8 types. Tranches and classes are defined by means of Roemer's Identification Assumption (RIA). That is, tranches

\footnotetext{
${ }^{7}$ For details, see Wagner, Frick and Schupp (2007).
} 
are defined by the quantile (we use deciles and ventiles in our empirical exercise) of the type-conditional distribution, whereas classes are defined by the quantile of the tranche-conditional distribution, with tranches ordered from rich to poor.

Our outcome variable is net labour income, expressed in real terms - at 2010 prices. ${ }^{8}$ As in previous studies, we select individuals aged 25 to 55 years. We examine three points in time: 2000, 2005 and 2010. After dropping observations with missing values for some of our circumstance variables or zero labour income, our sample size is 25,008 observations. Table 1 shows descriptive statistics of our sample, while Table 2 shows inequality trends over the sample period. In line with previous findings, income inequality increases over the whole sample period in Germany (Corneo, Zmerli and Pollak, 2014). The pattern of increasing labour income inequality is robust to using any of the three inequality measures we employed. In order to check whether inequality differences are statistically significant, we employ throughout the paper a standard test of means. Appendix Table A1 reports the results of such tests of means, and shows that such increase is statistically significant.

\subsection{Partial orderings}

The identification of effort with observed variables, such as own education attainment or hours worked, poses some problems on the implementation of the non anonymous ex post criteria. At each step of the sequential dominance test, this criterion entails comparing the outcomes (e.g. labor incomes) of the individuals located at the same point of the conditional effort distribution (i.e. within class) of the two distributions, i.e. comparing outcomes of the two individuals with the lowest effort, of the two individuals with the second lowest effort, and so on. When tranches are identified by means of responsibility variables, a first empirical problem has to do with how to rank individuals by effort levels when effort is defined in terms of a set of responsibility variables that need not have a natural ordering. Suppose, for instance that effort is defined in terms of own level of education and hours worked. Does the pair (low education, many hours worked) represent higher or lower effort than the pair (high education, few hours worked)? A second empirical problem comes from the fact that many individuals will have exactly the same effort level, as they will have the same values of the responsibility attributes, e.g. there will be many individuals in our sample with high level of education and many hours worked.

When tranches are identified by means of Roemer's Identification Assumption (RIA), we do not face the above problems. ${ }^{9}$ This is why in this empirical application we identify effort by means of RIA. This decision, however, comes at a cost. As pointed out in Section 3, in this case the non anonymous ex post criteria cannot be distinguished from the anonymous ex post criteria, since effort is defined according to the income position within types, and therefore

\footnotetext{
${ }^{8}$ We have used the consumer price index to deflate labour incomes.

${ }^{9}$ Now we can compare individuals located at the same position of the conditional effort distribution, and not two individuals will have the same degree of effort, as this is defined as percentiles of the conditional effort distribution.
} 
the ordering of individuals according to effort is the same as that according to labour income.

We will thus implement the other three ex-post criteria characterized in Section 5, and refer to the indistinguishable anonymous and non anonymous ex post criteria as "[non]anonymous". Before turning to the results, we would like to note that the sequential dominance tests required in the [non]anonymous and the egalitarian ex post criteria are not standard. They require adding up the incomes at the same effort level (percentile of the class distribution) of all classes involved. That is, at the first stage, we compare the labour income distribution of the first class for the two distributions involved. At the second stage, however, we compare the distributions that result from adding up the labour income of the individuals sitting on the same percentile of the first two classes. At the third stage, we have to proceed the same way for the first three classes, and so on until we add up all classes.

Table 3 displays the results from checking the (sequential) dominance conditions of the [non]anonymous, egalitarian, and utilitarian ex post criteria defined above. Absence of (sequential) dominance conditions is indicated by 'A', while (sequential) dominance of year c over year $\mathrm{r}$ is indicated by ' $<$ ', in cell $(\mathrm{r}, \mathrm{c})$. As panel (a) shows, the most restrictive criterion, the [non]anonymous ex post, is inconclusive about the ordering of the three distributions, as curves cross for each and every stage of the first order sequential dominance test. The egalitarian ex post criterion, however, yields statistically significant dominance results for two comparisons - see panel (b). In particular, we find that the 2000 distribution dominates both the 2005 and the 2010 distributions, but we cannot conclude anything about the ordering of the 2005 and 2010 distributions, as we have absence of sequential stochastic dominance. Of course, the utilitarian ex-post criterion yields the same results.

\subsection{Complete orderings}

We now turn to the opportunity inequality indices defined in Section 6 . We would first like to check whether the class approach put forth in the previous sections matters empirically. We thus compare our class approach with the related (ex-post) tranche and (ex-ante) type approach of Checchi and Peragine (2010). In a second exercise we use our class approach to examine inequality of opportunity in Germany over time, and compare the time trend of the class approach to the time trend of the (ex-ante) type and (ex-post) tranche approaches.

\subsubsection{The class approach as compared to the type and tranche ap- proaches.}

First we use data for year 2000 only, and compare the three approaches and the robustness of our findings to increasing the number of classes and tranches. We

employ three indices to estimate inequality: the MLD and two Atkinson indices with aversion parameters equal to 0.5 and 1 , and present our estimates in levels 
- in the upper panel of Tables 4 and 5- and relative to overall inequality -in the bottom panel of each Table. Since Atkinson indices do not decompose neatly in two additive components, but have a third multiplicative component, i.e. the product of the index of ex post equality of opportunity with the index of ex post inequality of effort, IO*IE, we provide an upper and a lower bound estimate of the relative effect of equality of opportunity. The lower bound estimate, shown in the upper part of each row, sets the interaction term of the decomposition to zero (i.e. $\mathrm{IO}^{*} \mathrm{IE}=0$ ), while the upper bound estimate, shown in brackets, considers the entire interaction term as part of inequality of opportunity, i.e. we report $\left(\mathrm{IO}+\mathrm{IO}^{*} \mathrm{IE} / \mathrm{I}\right)$.

The first three rows of the upper panel of Table 4 show that our ex post class approach yields opportunity inequality estimates that differ from those obtained either from the ex post tranche or the ex ante type approach. The class approach, however, does not yield systematically lower or higher levels of inequality of opportunity than the other two approaches, as this seems to depend on the inequality index used. While the class approach estimate lies in between the type and the tranche approach estimates for the MLD and the A(0.5), it yields the largest opportunity inequality estimate of the three approaches when A(1) is used. ${ }^{10}$ Appendix Table A2 shows that this pattern is statistically significant and holds also for years 2005 and 2010. Moreover, this pattern remains when we assess opportunity inequality relative to outcome inequality and is also robust to counting (or not counting) the multiplicative component of the Atkinson indices as opportunity inequality, as the first three rows of the bottom panel show.

The reaction to changing the number of partitions (classes or tranches) may differ between the two ex post approaches, as the tranche approach measures inequality within tranches, while the class approach measures inequality between classes. The tranche approach will most likely yield lower estimates of inequality of opportunity when the number of tranches increases and we use an additive decomposable index, such as the MLD. Since the tranche approach measures inequality within tranches, as we use finer partitions inequality decreases, unless the partitions have the same mean income - in which case, inequality remains the same irrespective of the number of partitions. For the class approach, however, it is difficult to sign a priori the inequality change resulting from finer partitions, as the class approach measures inequality between classes.

The second three rows of Table 4 help us assess how the two ex post approaches behave when the number of classes and/or tranches is increased (doubled). As expected, when we double the number of tranches from 10 to 20, the tranche approach yields a somewhat lower estimate of inequality of opportunity (compare rows 3 and 6 in Table 4). The class approach, however, behaves in the opposite direction when the number of classes doubles also from 10 to 20 , as inequality of opportunity with 20 classes is slightly larger than with 10 classes (compare rows 1 and 4 in Table 4). Appendix Table A4 shows that these

\footnotetext{
${ }^{10}$ The class approach also yields the largest inequality of opportunity estimate for Atkinson indices with parameter greater than 1.
} 
differences are statistically significant.

Now with 20 tranches and 20 classes, the class approach continues laying in between the type and the tranche approaches when inequality is measured with the MLD and the $\mathrm{A}(0.5)$ : When the $\mathrm{A}(1)$ is used, the class approach yields the largest estimate (compare now rows 2, 5, and 6). Appendix Table A5 show that these differences are statistically significant.

In sum, the class approach does yield different point estimates of inequality of opportunity than the other two approaches, the ex ante type and the ex post tranche approaches, both in absolute levels and as percentage of outcome inequality. However, the ordering of the three approaches crucially depends on the number of types, tranches, and classes one is working with as well as the index used to measure inequality. The class approach also behaves differently than the other ex post approach to changes in the number of partitions.

\subsubsection{Inequality of Opportunity over time in Germany.}

In spite of the differences between the three approaches at a point in time reported in Table 4, Table 5 shows that over time all approaches (and all three inequality indices, with only two exceptions ${ }^{11}$ ) order the three distributions the same way. There is larger inequality of opportunity in 2005, followed by 2010 and 2000. Notice that this result is consistent with our partial orderings results reported above.

The bottom panel of Table 5 shows that contrary to the observed trend in overall labour income inequality, over the first decade of the century, inequality of opportunity accounts for a smaller share of overall inequality.

\section{Concluding remarks}

In this paper we have proposed new ex post opportunity egalitarian criteria which, different from existing ones, explicitly recognize the interplay between circumstances and effort. We have characterized them axiomatically and we have also proposed some scalar measures which are inspired by such criteria. We have shown that our ex post criteria are mostly obtained from "seemingly" ex ante properties.

Then we have applied our new criteria to measuring inequality of opportunity in Germany, by using the German Socio-Economic Panel (SOEP) for the first decade of the 2000s.

Our results show that the new approach does yield different point estimates of inequality of opportunity with respect to the existing ex ante and the ex post approaches, both in absolute levels and as percentage of outcome inequality. However, the ordering of the three approaches crucially depends on the number of types, tranches, and classes. The class approach also reacts differently than the other ex post approach to changes in the number of partitions.

\footnotetext{
${ }^{11}$ The two exceptions have to do with the type approach, when using A(1) and MLD - the small difference in point MLD estimates is not statistically significant (see Table A3).
} 
We trust that the new tools introduced in this paper can help to shed new light on the analysis of opportunity inequality in different countries and different context. The paper opens new routes of research. From a theoretical perspective, the next step would consist in characterizing axiomatically the scalar measures proposed in the paper. From the empirical perspective a richer cross countries empirical investigation could help to appreciate the comparative advantage of our proposed approach with respect to the existing ones. These extensions are left for future research. 


\section{References}

Aaberge, R., Mogstad, M., Peragine, V. (2011). Measuring Long-term Inequality of Opportunity, Journal of Public Economics, 95, 193-204.

Bjorklund, A., Jantti, M., Roemer, J. (2011). Equality of opportunity and the distribution of long-run income in Sweden, Social Choice and Welfare, 1-22.

Bourguignon, F., Ferreira, F.H.G., Menéndez, M. (2007). Inequality Of Opportunity In Brazil, Review of Income and Wealth, 53(4), 585-18.

Brunori, P., Ferreira, F.H.G., Peragine, V. (2013), "Inequality of opportunity, income inequality and mobility: some international comparisons", in Paus E. ed. Getting Development Right: Structural Transformation, Inclusion and Sustainability in the Post-Crisis Era, Palgrave Macmillan.

Checchi, D., Peragine, V. (2010). Inequality of Opportunity in Italy, Journal of Economic Inequality, 8(4), 429-"50.

Checchi D., V.Peragine and L.Serlenga. 2010. Fair and Unfair Income Inequalities in Europe. IZA Discussion Paper No. 5025/2010

Ferreira, F.H.G., Gignoux, J. (2011). The measurement of inequality of opportunity: theory and an application to Latin America, Review of Income and Wealth, 57(4), 622-657.

Ferreira, Francisco H. G., and Vito Peragine. 2015. Individual Responsibility and Equality of opportunity. in M. Adler and M. Fleurbaey (eds), Handbook of Well Being and Public Policy, Oxford University Press, 2015.

Fleurbaey, M. (2008). Fairness, responsibility and welfare, Oxford University Press.

Fleurbaey, M., Peragine, V. (2013). Ex ante versus ex post equality of opportunity, Economica, 8(317), 118-30.

LeFranc, A., Pistolesi, N., Trannoy, A. (2008). Inequality Of Opportunities Vs. Inequality Of Outcomes: Are Western Societies All Alike? Review of Income and Wealth, 54(4), 513-46.

Peragine, V. (2002). Opportunity Egalitarianism and Income Inequality: the Rank-Dependent Approach, Mathematical Social Sciences, 44, 45-64.

Peragine, V., Palmisano, F., Brunori, P. (2014). Economic growth and equality of opportunity, World Bank Economic Review, 28(2), 247-281. 
Peragine, V., Serlenga, L. (2008). Higher Education and Equality of Opportunity in Italy, In J. Bishop, and B. Zheng, eds., Research in Economic Inequality, 16.

Ramos, X., Van de gaer, D. (2012). Empirical approaches to inequality of opportunity: Principles, Measures and Evidences, Working Paper 259. ECINEQ. Roemer J.E. 1993 A pragmatic theory of responsibility for the egalitarian planner. Philosophy and Public Affairs 22, 146-166.

Roemer, J.E. (1998). Equality of opportunity. Harvard University Press, Cambridge, MA.

Roemer, John, and Alain Trannoy. 2015. Equality of Opportunity. in Atkinson and F.Bourguignon (eds). Handbook of income distribution, vol.2B, North Holland

Van de Gaer, D. 1993. Equality of Opportunity and Investment in Human Capital, Ph.D. Dissertation, Katholieke Universiteit Leuven, Belgium.

Gert G. Wagner \& Joachim R. Frick \& Jürgen Schupp, 2007. "The German Socio-Economic Panel Study (SOEP) - Scope, Evolution and Enhancements," Schmollers Jahrbuch : Journal of Applied Social Science Studies / Zeitschrift für Wirtschafts- und Sozialwissenschaften, Duncker \& Humblot, Berlin, vol. 127(1), pages 139-169.

World Bank (2006). World Development Report 2006: equity and development, Washington, DC:World Bank. 


\section{Appendix}

\section{Ex-post inequality of opportunity in Germany}

Table 1. Descriptive statistics of our sample

\begin{tabular}{|c|c|c|c|c|c|}
\hline Variable & $\mathrm{N}$ & Mean & Std. Dev. & Min & Max \\
\hline \multicolumn{6}{|c|}{ Year 2000} \\
\hline Labor income & 9325 & 1657.83 & 1004.08 & 23.94 & 14966.69 \\
\hline Men & 9325 & 0.56 & 0.50 & 0 & 1 \\
\hline Older than 40 & 9325 & 0.50 & 0.50 & 0 & 1 \\
\hline Parental education: secondary or more & 9325 & 0.31 & 0.46 & 0 & 1 \\
\hline \multicolumn{6}{|c|}{ Year 2005} \\
\hline Labor income & 8429 & 1818.57 & 1371.16 & 1.09 & 21729.79 \\
\hline Men & 8429 & 0.52 & 0.50 & 0 & 1 \\
\hline Older than 40 & 8429 & 0.58 & 0.49 & 0 & 1 \\
\hline Parental education: secondary or more & 8429 & 0.39 & 0.49 & 0 & 1 \\
\hline \multicolumn{6}{|c|}{ Year 2010} \\
\hline Labor income & 7254 & 1741.27 & 1300.27 & 8 & 20000 \\
\hline Men & 7254 & 0.50 & 0.50 & 0 & 1 \\
\hline Older than 40 & 7254 & 0.64 & 0.48 & 0 & 1 \\
\hline Parental education: secondary or more & 7254 & 0.45 & 0.50 & 0 & 1 \\
\hline
\end{tabular}

Source: own calculations from SOEP.

Table 2. Labour income inequality over the 2000s.

\begin{tabular}{lccc}
\hline & \multicolumn{3}{c}{ Labour income } \\
\hline & 2000 & 2005 & 2010 \\
\hline MLD & 0.193 & 0.246 & 0.255 \\
Standard Error & {$[.0002]$} & {$[.0004]$} & {$[.0003]$} \\
Atkinson (0.5) & 0.085 & 0.108 & 0.111 \\
Standard Error & {$[.0001]$} & {$[.0002]$} & {$[.0002]$} \\
Atkinson (1) & 0.175 & 0.218 & 0.225 \\
Standard Error & {$[.0002]$} & {$[.0003]$} & {$[.0003]$} \\
\hline
\end{tabular}

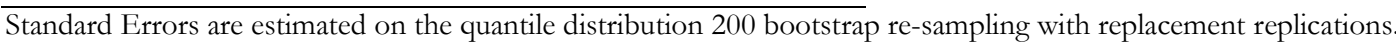
Source: own calculations from SOEP. 
Table 3. (sequential) Dominance test for S-EOP, A-EOP, E-EOP, U-EOP.

Germany, 2000, 2005, 2010.

\begin{tabular}{ccc}
\hline \hline \multicolumn{3}{c}{ (a) [Non] Anonymous (S-EOP, A-EOP) } \\
\hline & 2000 & 2005 \\
2005 & $\mathrm{~A}$ & \\
2010 & $\mathrm{~A}$ & $\mathrm{~A}$ \\
\cline { 2 - 3 }
\end{tabular}

\begin{tabular}{ccc}
\hline \hline (b) Egalitarian (E-EOP) \\
\hline & 2000 & 2005 \\
\cline { 2 - 3 } 2005 & $<^{*}$ & \\
2010 & $<^{*}$ & A
\end{tabular}

\begin{tabular}{|c|c|c|}
\hline \multicolumn{3}{|c|}{ (c) Utilitarian (U-EOP) } \\
\hline & 2000 & 2005 \\
\hline 2005 & $<^{*}$ & \\
\hline 2010 & $<*$ & A \\
\hline
\end{tabular}

$\overline{\text { A denotes absence of stochastic dominance, }} \overline{\overline{\text { while ' }<\text { ' denotes that year } c}}$ dominates year $r$ in cell $(r, c)$. $*$ denotes that the dominance test is statistically significant at $5 \%$.

Confidence intervals are estimated on the quantile distribution 200 bootstrap re-sampling with replacement replications.

Source: own calculations from SOEP.

Table 4. Class, Tranche, and Type Approaches to Inequality of Opportunity in Germany, 2000

\begin{tabular}{|c|c|c|c|c|c|c|}
\hline Approach & MLD & $\mathrm{A}(0.5)$ & $\mathbf{A}(1)$ & \# Classes & \#Tranches & \#Types \\
\hline \multicolumn{7}{|c|}{ Levels } \\
\hline Class & 0.0527 & 0.0279 & 0.0648 & \multirow[t]{6}{*}{10} & \multirow[t]{4}{*}{10} & 8 \\
\hline Standard Error & {$[.0017]$} & {$[.0008]$} & {$[.0020]$} & & & \\
\hline Type & 0.0469 & 0.0253 & 0.0583 & & & 8 \\
\hline Standard Error & {$[.0016]$} & {$[.0008]$} & {$[.0019]$} & & & \\
\hline Tranche & 0.0793 & 0.0297 & 0.0593 & & \multirow[t]{2}{*}{10} & \multirow[t]{2}{*}{8} \\
\hline Standard Error & {$[.0013]$} & {$[.0006]$} & {$[.0010]$} & & & \\
\hline Class & 0.0537 & 0.0285 & 0.0659 & \multirow[t]{2}{*}{20} & \multirow[t]{2}{*}{10} & \multirow[t]{2}{*}{8} \\
\hline Standard Error & {$[.0018]$} & {$[.0009]$} & {$[.0021]$} & & & \\
\hline Class & 0.0504 & 0.0269 & 0.0621 & \multirow[t]{4}{*}{20} & 20 & \multirow[t]{2}{*}{8} \\
\hline Standard Error & {$[.0019]$} & {$[.0010]$} & {$[.0022]$} & & \multirow{3}{*}{20} & \\
\hline Tranche & 0.0739 & 0.0276 & 0.0551 & & & \multirow[t]{2}{*}{8} \\
\hline Standard Error & {$[.0011]$} & {$[.0004]$} & {$[.0008]$} & & & \\
\hline \multicolumn{7}{|c|}{$\%$ Overall Inequality } \\
\hline \multirow[t]{2}{*}{ Class } & 0.274 & 0.324 & 0.354 & \multirow[t]{6}{*}{10} & \multirow[t]{4}{*}{10} & \multirow[t]{2}{*}{8} \\
\hline & & {$[0.350]$} & {$[0.414]$} & & & \\
\hline \multirow[t]{2}{*}{ Type } & 0.243 & 0.294 & 0.320 & & & \multirow[t]{2}{*}{8} \\
\hline & & {$[0.318]$} & {$[0.374]$} & & & \\
\hline \multirow{2}{*}{ Tranche } & 0.412 & 0.344 & 0.325 & & \multirow[t]{2}{*}{10} & \multirow[t]{2}{*}{8} \\
\hline & & {$[0.371]$} & {$[0.338]$} & & & \\
\hline \multirow[t]{2}{*}{ Class } & 0.279 & 0.331 & 0.361 & \multirow[t]{2}{*}{20} & \multirow[t]{2}{*}{10} & \multirow[t]{2}{*}{8} \\
\hline & & {$[0.357]$} & {$[0.420]$} & & & \\
\hline \multirow[t]{2}{*}{ Class } & 0.262 & 0.312 & 0.340 & \multirow[t]{4}{*}{20} & \multirow[t]{2}{*}{20} & \multirow[t]{2}{*}{8} \\
\hline & & {$[0.337]$} & {$[0.397]$} & & & \\
\hline \multirow[t]{2}{*}{ Tranche } & 0.384 & 0.320 & 0.303 & & \multirow[t]{2}{*}{20} & 8 \\
\hline & & {$[0.345]$} & {$[0.355]$} & & & \\
\hline
\end{tabular}

Note: Tranches are identified by means of RIA.

Upper panel shows in brackets standard errors estimated on the quantile distribution 200 bootstrap re-sampling with replacement replications.

Lower panel shows in brackets the upper bound estimate of the inequality of opportunity share over outcome inequality.

Source: own calculations from SOEP. 
Table 5. Class, Tranche, and Type Approaches to Inequality of Opportunity in Germany over time, 2000, 2005, 2010.

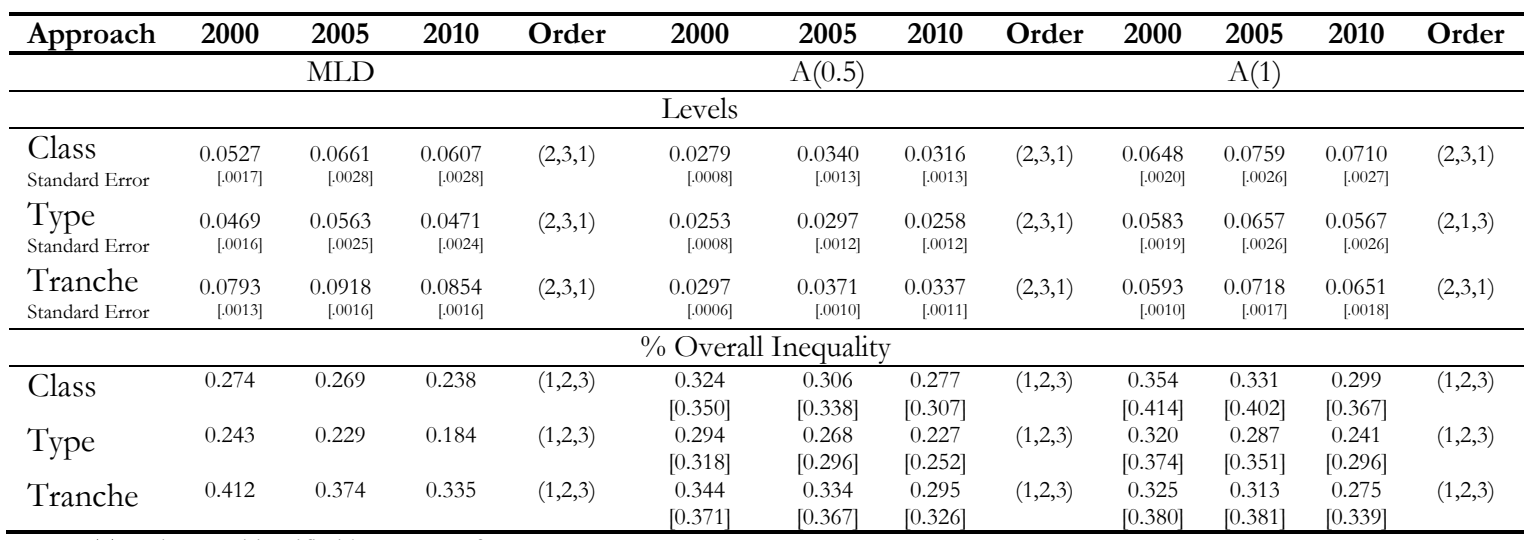

Note: Tranches are identified by means of RIA.

Upper panel shows in brackets standard errors estimated on the quantile distribution 200 bootstrap re-sampling with replacement replications.

Lower panel shows in brackets the upper bound estimate of the inequality of opportunity share over outcome inequality. Source: own calculations from SOEP. 


\section{Appendix: Are inequality differences over time/across approaches statistically significant?}

Table A1. Are inequality differences over time statistically significant? Germany, 2000, 2005, 2010.

\begin{tabular}{|c|c|c|c|c|c|c|c|c|}
\hline \multicolumn{3}{|c|}{ (a) MLD } & \multicolumn{3}{|c|}{ (b) $\mathrm{A}(0.5)$} & \multicolumn{3}{|c|}{ (c) $\mathrm{A}(1)$} \\
\hline & 2000 & 2005 & & 2000 & 2005 & & 2000 & 2005 \\
\hline 2005 & $>^{*}$ & & 2005 & $>^{*}$ & & 2005 & $>^{*}$ & \\
\hline 2010 & $>^{*}$ & $>^{*}$ & 2010 & $>^{*}$ & $>^{*}$ & 2010 & $>^{*}$ & $>^{*}$ \\
\hline
\end{tabular}

Source: own calculations from SOEP.

Table A2. Are Inequality of Opportunity differences across approaches statistically significant? Germany, 2000, 2005, 2010.

\begin{tabular}{|c|c|c|c|c|c|c|c|c|}
\hline & & & & ar 2000 & & & & \\
\hline & MLD & & & $\begin{array}{c}\mathrm{A}(0.5) \\
\end{array}$ & & & $\overline{\mathrm{A}(1)}$ & \\
\hline & Class & Type & & Class & Type & & Class & Type \\
\hline Type & $<^{*}$ & & Type & $<^{*}$ & & Type & $<^{*}$ & \\
\hline Tranche & $>^{*}$ & $>^{*}$ & Tranche & $>^{*}$ & $>^{*}$ & Tranche & $<^{*}$ & $<^{*}$ \\
\hline & & & & ar 2005 & & & & \\
\hline & MLD & & & $\bar{A}(0.5)$ & & & $\overline{\mathrm{A}(1)}$ & \\
\hline & Class & Type & & Class & Type & & Class & Type \\
\hline Type & $<^{*}$ & & Type & $<^{*}$ & & Type & $<^{*}$ & \\
\hline Tranche & $>^{*}$ & $>^{*}$ & Tranche & $>^{*}$ & $>^{*}$ & Tranche & $<*$ & $<*$ \\
\hline & & & & $\operatorname{ar} 2010$ & & & & \\
\hline & MLD & & & $\overline{\mathrm{A}(0.5)}$ & & & $\overline{\mathrm{A}(1)}$ & \\
\hline & Class & Type & & Class & Type & & Class & Type \\
\hline Type & $<^{*}$ & & Type & $<^{*}$ & & Type & $<^{*}$ & \\
\hline Tranche & $>^{*}$ & $>^{*}$ & Tranche & $>^{*}$ & $>^{*}$ & Tranche & $<^{*}$ & $<*$ \\
\hline $\begin{array}{l}* \text { denotes } \\
\text { that opp } \\
\text { inequality }\end{array}$ & $\begin{array}{l}\text { hat it } \\
\text { tunit } \\
\text { ccor }\end{array}$ & $\begin{array}{l}\text { uality } \\
\text { nequa } \\
\text { g to a }\end{array}$ & $\begin{array}{l}\text { erence is } \\
\text { according } \\
\text { ach } r(c)\end{array}$ & $\begin{array}{l}\overline{\text { tistic }} \\
\text { o apl } \\
\text { cell }\end{array}$ & $\begin{array}{l}\text { lly sig1 } \\
\text { oach }\end{array}$ & $\begin{array}{l}\text { at at } 1 \% \\
\text { s larger }\end{array}$ & $\begin{array}{l}\bar{R}^{\prime} \text { ('> } \\
\text { an of }\end{array}$ & $\begin{array}{l}\text { denot } \\
\text { ortuni }\end{array}$ \\
\hline Source: $\mathrm{O}$ & n calc & ulations & m SOEP & & & & & \\
\hline
\end{tabular}


